\title{
Effets d'une augmentation de la fumure azotée ou de la distribution supplémentaire de concentré sur les performances et les teneurs en urée plasmatique de vaches laitières soumises au pâturage continu et en rotation *
}

\author{
I Dufrasne 1, M Gielen 1, P Limbourg 2, N Korsak 1, L Istasse ${ }^{1}$ \\ ${ }^{1}$ Service de nutrition, faculté de médecine vétérinaire, université de Liège, \\ B43 Sart Tilman, 4000 Liège; \\ 2 Centre de recherche agronomique, rue du Serpont 100, 6800 Libramont, Belgique
}

(Reçu le 8 août 1994 ; accepté le 22 août 1995)

\begin{abstract}
Résumé - Un essai a été réalisé avec des vaches laitières au pâturage vêlées au printemps afin d'aug. menter la production par hectare grâce à l'augmentation conjointe du chargement et du niveau de concentré ou d'engrais azoté dans le système de pâturage continu ou en rotation. L'essai a été répété au cours de 2 années consécutives. Trente-quatre vaches ont été réparties en six traitements, les six traitements correspondaient à deux systèmes de conduite de pâturage : rationné $(1,2,3)$ ou continu $(4,5,6)$. Dans chaque système un traitement était conduit avec un chargement de quatre vaches par hectare et deux traitements avec cinq vaches par hectare. L'accroissement du chargement a été obtenu par l'accroissement du niveau de complémentation $(2,5)$ ou par celui de la fertilisation azotée $(3,6)$. L'apport supplémentaire de concentré a été calculé de manière à couvrir les besoins d'une vache ayant la production moyenne du lot. L'accroissement de la fertilisation avait pour objectif de maintenir une même quantité d'herbe offerte par vache entre les traitements 3 et 6 et 1 et 4 en admettant que $1 \mathrm{~kg}$ d'engrais azoté apportait 1,3 jour de pâturage. Finalement les animaux ont reçu 1,$4 ; 1,3$; 4,$2 ; 3,9 ; 1,2$ et $1,1 \mathrm{~kg}$ de concentré dans les traitements $1,4,2,5,3$ et 6 respectivement et les niveaux de fumure ont été de $113 \mathrm{~kg}$ d'engrais azoté par hectare pour les traitements $1,2,4$ et 5 et de $225 \mathrm{~kg}$ d'engrais azoté par ha pour les traitements 3 et 6 . Chaque année, cinq vaches ont été utilisées dans les traitements 1 et 4 et six vaches dans chacun des autres traitements. La production de la prairie a été augmentée de $1435 \mathrm{~kg}$ de matière sèche dans les traitements 3 et 6 par rapport aux traitements 1 et 4 et la proportion de trèfle réduite de 12 à $8 \%$. Les teneurs en matière sèche, cellulose brute et matières azotées n'ont pas été influencées par les traitements $(16,3 \%$ de matière sèche, $20,2 \%$ de cellulose brute, $23,8 \%$ de matières azotées). Les productions laitières des traitements 2 et 5 ont été supérieures à celles des traitements 1 et $4(15,61$ versus $14,56 \mathrm{~kg} / \mathrm{jour} ; p>0,10)$ tandis que, dans les traitements 3 et 6 , elles ont été inférieures à celles des traitements 1 et $4(13,12$ versus $14,56 \mathrm{~kg} /$ jour $; p>0,10$ ). II n'y a pas eu d'interaction entre le système de pâturage et les différentes moda-

* Travail financé par l'Irsia (Institut pour l'encouragement de la recherche scientifique dans l'industrie et l'agriculture).
\end{abstract}


lités. La productivité de la prairie, exprimée en UFL/ha, a été plus élevée dans les traitements 3 et 6 que dans les traitements 1 et 4 (8168 versus 7159 UFL/ha) et dans le système de pâturage en rotation que dans le pâturage continu ( 7650 versus 7309 UFL/ha). Les teneurs en urée ont été réduites dans les traitements 2 et 5 par rapport aux traitements 1 et 4 ( 218 versus $231 \mathrm{mg} \mathrm{N} / \mathrm{L} ; p>0,10)$. Dans les traitements 3 et 6 , elles ont été plus élevées ( 269 versus $231 \mathrm{mg} \mathrm{N} / L ; p<0,001$ ). Dans le pâturage en rotation, elles ont été inférieures à celles du pâturage continu (209 versus $270 \mathrm{mg} \mathrm{N} / \mathrm{L} ; p<$ $0,001)$. Il y a eu une interaction significative entre le système de pâturage et les différentes modalités.

\section{vaches laitières / chargement / complément / azote}

Summary - Effects of an increase in nitrogen fertilizer or in supplementary concentrate on performances and on plasma urea with lactating dairy cows grazed either continuously or in a rotational system. A grazing trial was conducted with spring calving dairy cows grazed either continuously or with the rotational system in order to improve yield per ha with a simultaneous increase in stocking rate and either in concentrate or in nitrogen fertilizer. The trial was repeated over 2 consecutive years. There were 34 cows divided into six treatment groups: groups 1,2 and 3 were grazed with the rotational system and groups 4,5 and 6 were grazed continuously. Three methods of grazing were used in each system. In the first method of grazing, the stocking rate was four cows/ha (groups 1 and 4). In the two other methods of grazing, the stocking rate was five cows/ha: the increase in stocking rate was allowed by an increase in the amount of concentrate (groups 2 and 5) or by an increase in nitrogen fertilizer (groups 3 and 6). The supplementary concentrate offered in groups 2 and 5 as compared with groups 1 and 4 was calculated to cover the requirements of the supplementary cow with the average milk yield of the group. In groups 3 and 6 , the amount of nitrogen fertilizer was increased in order to compensate for the increase of stocking rate, which reduced the grazing area per cow, by an improvement in grass production induced by supplementary nitrogen fertilizer, $1 \mathrm{~kg}$ nitrogen fertilizer allowing 1.3 days extra grazing. Concentrate was offered to all the cows when they were milked. The cows received 1.41 and $1.28 \mathrm{~kg}$ concentrate daily in groups 1 and 4 , respectively. In groups 2 and 5 , the cows were offered a large amount of concentrate: $4.18 \mathrm{~kg} /$ day in group 2 and $3.89 \mathrm{~kg} /$ day in group 5 . The cows in group 3 ate $1.21 \mathrm{~kg}$ of concentrate and those in group $6,1.07 \mathrm{~kg}$. Nitrogen fertilizer was applied at the rate of $113 \mathrm{~kg} / \mathrm{ha}$ in the pasture grazed by the cows in groups 1 , 2,4 and 5 and at the rate of $225 \mathrm{~kg} \mathrm{~N} / \mathrm{ha}$ in the pasture grazed by the cows in groups 3 and 6 . Each year, five cows were utilized in groups 1 and 4 and six cows in the other groups. Grass production was improved by an extra $1435 \mathrm{~kg}$ dry matter in groups 3 and 6 as compared with groups 1 and 4 and the proportion of clover was reduced from 12 to $8 \%$. The contents in dry matter, crude fiber and crude protein of grass were not affected by the treatments, the corresponding figures being $16.3 \%, 20.1 \%$ and 23.8\%. The milk yields in groups 2 and 5 were higher than in groups 1 and 4 (15.61 vs $14.56 \mathrm{~kg} /$ day; $\mathrm{P}>0.10$ ) while in groups 3 and 6 they were lower than in groups 1 and 4 (13.12 vs $14.56 \mathrm{~kg} /$ day; $\mathrm{P}>$ 0.10 ). There were no interactions between grazing systems and methods of grazing. Grass yields, expressed in UFL/ha, were higher in groups 3 and 6 than in groups 1 and 4 (8 168 vs 7159 UFL/ha) and in the rotational system than in the continuous system ( 7650 vs 7309 UFLha). Urea concentrations in blood plasma were reduced in groups 2 and 5 as compared with groups 1 and 4 (218 vs 231 $\mathrm{mg} \mathrm{N} / \mathrm{P} \mathrm{P}>0.10$ ); in groups 3 and 6 , the concentrations were the highest (269 vs $231 \mathrm{mg} \mathrm{N} / \mathrm{P} \mathrm{P}<0.001$ ). The rotational system induced lower concentrations than the continuous system (209 vs $270 \mathrm{mg} \mathrm{N} /$; $\mathrm{P}<0.001)$. There was an interaction between the grazing systems and the methods of grazing.

dairy cows / stocking rate / supplement / nitrogen

\section{INTRODUCTION}

Depuis 1984, la production de lait dans les exploitations est limitée par un système de quota. Dans des régions où la surface agri- cole est limitée, la production de lait à partir de l'herbe pâturée reste intensive. L'augmentation du chargement représente un moyen d'augmenter la production par hectare, mais diminue généralement la pro- 
duction par animal (Béranger et Micol, 1981 ; Hoden et al, 1991). Il est cependant possible d'obtenir des performances semblables lorsque l'augmentation du chargement s'accompagne d'une augmentation du niveau de concentré ou de la fumure azotée, comme l'ont prouvé Limbourg et al (1980) dans un pâturage en rotation. Le maintien des performances est lié au fait que la distribution de concentré au pâturage permet d'augmenter la quantité de matière organique ingérée (Kibon et Holmes, 1987) et qu'un accroissement d'engrais azoté permet d'augmenter la production d'herbe et donc les quantités d'herbe offertes.

L'augmentation de la fumure azotée accroît de façon linéaire le nombre de jours de pâturage (Hodgson, 1990) et de façon curvilinéaire la potentialité de chargement (Gately et O'Keeffe, 1984). La fumure azotée augmente la teneur en matières azotées des graminées (Demarquilly, 1977) et la fraction azotée non protéique (Goswami et Willcox, 1969), ce qui se répercute par un accroissement de la dégradabilité des matières azotées de l'herbe dans le rumen (Van Vuuren et al, 1991) et par un surplus d'azote fermentescible (Van Vuuren et al, 1990). II peut en résulter une augmentation de la teneur en urée dans le plasma sanguin et dans le lait, suite à l'étroite corrélation entre ces paramètres (Oltner et Wiktorsson, 1983 ; Miettinen et Juvonen, 1990). Or, la présence d'urée dans le lait conditionne son aptitude à la stérilisation (Mottar et De Vilder, 1979) et son rendement fromager. En outre, la teneur en urée du lait permet une estimation de l'excrétion d'azote urinaire chez les vaches (Ciszuk et Gebregziabher, 1994).

L'efficacité du concentré distribué au pâturage est de l'ordre de $0,4 \mathrm{~kg}$ de lait $/ \mathrm{kg}$ de concentré (Journet et Demarquilly, 1979), mais la réponse de la production laitière par kilo de lait peut être augmentée si le niveau d'ingestion d'herbe diminue (Stockdale et Trigg, 1989). De plus, les rejets azotés peuvent être diminués par la distribution de concentré (Van Vuuren, 1993), ce qui peut être particulièrement intéressant car les facteurs environnementaux seront pris de plus en plus en considération à l'avenir dans le cadre de la politique agricole commune.

Le système de pâturage, pâturage continu ou en rotation, peut également avoir une influence sur les performances des animaux. Hood (1974), Castle et Watson (1975), Mott et al (1980) et Schlepers et Lantinga (1985) n'ont observé que des différences minimes entre ces deux systèmes de pâturage. En revanche, Chopping et al (1978) ont noté une amélioration de la production de l'ordre de $10 \%$ en faveur du système en rotation tandis que le pâturage continu a été bénéfique dans l'essai de Lebrun (1987) lorsque le niveau de complémentation était plus élevé. En Belgique, Carlier et Andries (1981) ont mis en évidence une augmentation des productions de 3 à $4 \%$ grâce au système en rotation lorsque les fumures azotées étaient supérieures à $400 \mathrm{~kg} \mathrm{~N} / \mathrm{ha}$. Bienfait et al (1981) et Gielen et al (1983) ont obtenu des performances semblables entre les deux systèmes chez des vaches laitières avec une fumure azotée plus élevée dans le pâturage continu.

L'objectif de cet essai est d'étudier les possibilités d'augmenter le chargement sans réduire les productions individuelles en utilisant un haut niveau de concentré ou d'engrais azoté dans un système de pâturage continu ou en rotation. Les répercussions de ces différents traitements sur les teneurs en urée plasmatique des vaches et sur la production de l'herbe et la disponibilité en herbe ont été étudiées.

\section{MATÉRIEL ET MÉTHODES}

\section{Animaux et conduite du pâturage}

L'essai a été réalisé pendant 2 années consécutives sur des prairies de longue durée situées 
dans les Ardennes belges. Chaque année, l'essai a commencé après une période de transition de 10 jours pendant la deuxième décade de mai et s'est terminé pendant la deuxième décade d'octobre, soit après 140 jours de pâturage. Le schéma expérimental est indiqué dans le tableau I. Un total de 34 vaches Pie-Noir ayant vêlé début mars a été utilisé chaque année. Ces 34 vaches ont été réparties en six groupes homogènes basés sur le numéro de lactation, la date de vêlage et la production laitière. Les animaux des traitements 1,2 et 3 ont pâturé dans un système en rotation basé sur six parcelles tandis que les vaches des traitements 4,5 et 6 pâturaient dans un système continu. Dans chaque système, trois modalités de pâturage ont été testées : un chargement de quatre vaches/ha dans les traitements 1 et 4 , un chargement de cinq vaches/ha avec niveau élevé de concentré dans les traitements 2 et 5 et un chargement de 5 vaches/ha avec un niveau élevé d'engrais azoté dans les traitements 3 et 6 . Dans les traitements 1 et 4 , la superficie était de 1,25 ha et il y avait cinq vaches par traitement. Dans les quatre autres traitements, la surface était de 1,20 ha pour six animaux. Dans tous les traitements, les vaches ont reçu un complément constitué par un mélange de pulpes sèches de betteraves sucrières et d'orge aplatie en proportions égales, dont la composition chimique est la suivante : $87,3 \%$ de matière sèche et $4,9 \%$ de cendre, $16,9 \%$ de cellulose brute, $11,8 \%$ de matières azotées totales, $1,3 \%$ d'extrait éthéré dans la matière sèche. La flore des prairies, estimée par une méthode adaptée de la méthode analytique des fréquences (Andries, 1950), était constituée de ray-grass anglais (35-45\%), de pâturin commun $(7-10 \%)$, de pâturin des prés $(5-7 \%)$, de dactyle et de fléole $(2-5 \%)$ et de trèfle blanc $(5-15 \%)$. On y retrouvait également du pissenlit (8-9\%). Lors de la mise en lots, les vaches pesaient en moyenne $485 \mathrm{~kg}$ et avaient vêlé depuis 55 jours. Leur numéro moyen de vêlage était égal à 3,06. La production laitière initiale était de $20,7 \mathrm{~kg}$ par vache en année 1 et de 19,8 $\mathrm{kg}$ par vache en année 2. Dans les traitements $1,3,4$ et 6 , le concentré était distribué à raison de $1 \mathrm{~kg}$ par tranche de $2,5 \mathrm{~kg}$ de lait pour les productions supérieures à $11 \mathrm{~kg} / \mathrm{jour}$. L'ajustement des apports en concentré a été établi grâce aux données bimensuelles de productions laitières et aux pesées mensuelles des animaux. Dans les traitements 2 et 5, l'augmentation du chargement qui réduit la surface en herbe par animal est compensée par une augmentation de la distribution individuelle d'aliment concentré. Cette quantité d'aliment supplémentaire a été calculée de manière à couvrir les besoins d'une vache dont le poids et la production correspondaient à la moyenne du lot. Les besoins d'entretien, de production laitière et de modification de poids ont été estimés à partir des données de Vérité et al (1980). L'engrais azoté a été apporté en plusieurs fractions au cours de la période de pâturage sous forme de nitrate d'ammonium au même moment dans les six traitements. Les traitements 3 et 6 à fumure élevée ont reçu des quantités d'engrais deux fois plus élevées à chaque application. L'augmentation du niveau de fumure azotée dans les traitements 3 et 6 par rapport aux traitements 1 et 4 a été calculée selon les résultats de Limbourg et al (1980) où chaque kilo d'engrais azoté permettait 1,30 jour de pâturage supplémentaire. Pour obtenir une augmentation de 140 jours de pâturage, soit l'augmentation de chargement d'une vache par hectare, un accroissement de

Tableau I. Schéma expérimental.

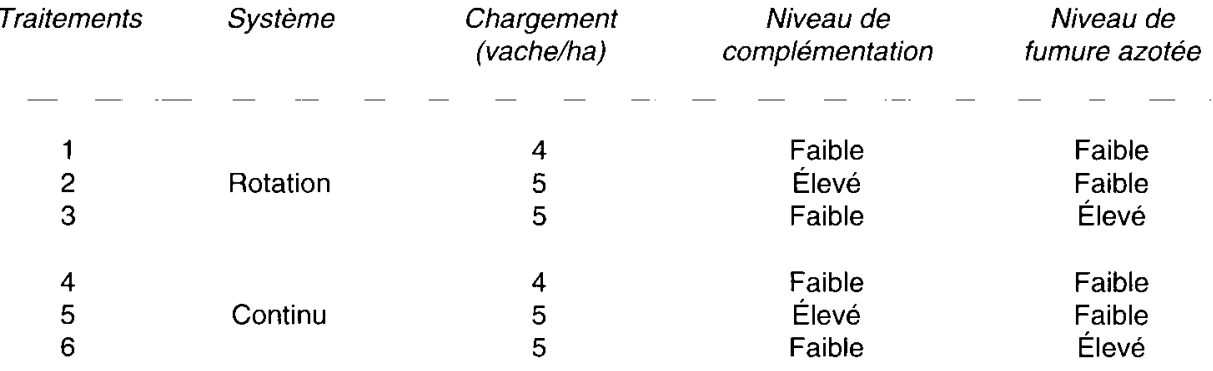


la fumure azotée de $110 \mathrm{~kg}$ était nécessaire. Les quantités d'engrais azoté appliqué ont légèrement varié d'une année à l'autre selon les conditions climatiques. Elles ont été de 100 et $200 \mathrm{~kg} / \mathrm{ha}$ pendant l'année 1 et de 125 et de $250 \mathrm{~kg} / \mathrm{ha}$ pendant l'année 2 dans les chaînes à fumure modérée et élevée respectivement. Au printemps, l'excédent d'herbe a été valorisé par un troupeau de génisses hors essai dont les journées de pâturage ont été comptabilisées. Dans le système en rotation, la sortie des animaux des parcelles a été décidée selon la disponibilité en herbe et lorsque la production laitière moyenne chutait de $2 \mathrm{~kg}$ par rapport à sa valeur maximale sur la parcelle. Les durées du cycle de rotation et les durées de séjour sur les parcelles ont été en moyenne respectivement de 31 à 5,20 jours dans le traitement 1 , de 29 et 4,84 jours dans le traitement 2 et de 25 et 4,10 jours dans le traitement 3. Aucun ébousage n'a été pratiqué pendant la période expérimentale.

\section{Mesures phytotechniques}

Les mesures phytotechniques ont consisté en la détermination de la production primaire de la prairie, de la composition chimique de l'herbe, de la hauteur de l'herbe pâturée et de la composition floristique. Les mesures des productions primaires ont été réalisées à l'aide d'une tondeuse à gazon par coupe à une hauteur de $2,5 \mathrm{~cm}$ dans des enclos soustraits au pâturage. Dans le système continu, il y avait deux enclos par traitement dont la superficie à couper était de $21 \mathrm{~m}^{2}$. Les enclos étaient composés d'un damier de cellules récoltées chaque semaine grâce à un système tournant à des emplacements prédéterminés de façon à imiter le mode de prélèvement des animaux. Les repousses ainsi récoltées étaient âgées de 1 , 2 ou 3 semaines. Dans le pâturage en rotation, il $y$ avait trois enclos dont la superficie à couper est de $10 \mathrm{~m}^{2}$. La récolte était effectuée juste avant l'arrivée du bétail dans les parcelles. Les hauteurs d'herbe ont été estimées à l'aide d'un herbomètre à plateau. Le plateau était composé d'une feuille d'aluminium de $30 \mathrm{~cm}$ sur $30 \mathrm{~cm}$, exerçant une pression de $2 \mathrm{~kg} / \mathrm{m}^{2}$ et coulissant sur un axe gradué. Dans le pâturage continu, 66 mesures par hectare ont été réalisées chaque semaine. Dans le pâturage en rotation, les hauteurs étaient déterminées lors de l'entrée des animaux dans la parcelle en effectuant 200 mesures par hectare.
La composition chimique de l'herbe a été déterminée à partir d'échantillons prélevés aux ciseaux à une hauteur de $2,5 \mathrm{~cm}$ dans le pâturage en rotation et de $1 \mathrm{~cm}$ dans le pâturage continu. Les hauteurs de coupe différentes selon le système de pâturage visent à obtenir des échantillons d'herbe se rapprochant le plus possible de l'herbe ingérée par les animaux. Dans le pâturage en rotation, les échantillons étaient prélevés lors de l'arrivée des animaux dans les parcelles. Dans le pâturage continu, les échantillons étaient prélevés toutes les semaines. Lors de chaque prélèvement, un échantillon correspondant à 10 poignées par traitement était collecté. Les échantillons ont ensuite été regroupés en vue de leur analyse par période de 4 semaines pour chaque traitement. Les teneurs en matières azotées et en cellulose brute ont été déterminées par spectrométrie dans le proche infrarouge (PSco 6250, Silver Spring, MD, États-Unis).

\section{Mesures zootechniques}

Les mesures zootechniques comprenaient la pesée des productions laitières individuelles lors de la traite du matin $(6 \mathrm{~h} 00)$ et du soir (15 h 00). Chaque semaine, un échantillon représentatif de la traite du matin et du soir a été prélevé et analysé afin de déterminer la composition en matière grasse et en matière azotée. Les animaux ont été pesés tous les 28 jours à la même heure (10 h 00). Pendant la deuxième année, un échantillon de sang a été prélevé au niveau de la veine jugulaire lors de chaque pesée. Après centrifugation, les plasmas ont été placés au congélateur. Ils ont ensuite été analysés pour déterminer la concentration en urée plasmatique par la méthode de la diacétylmonoxime (Wybenga et al, 1971, cité par Henry et al, 1974). La productivité de la prairie, exprimée en énergie nette (UFL/ha) a été calculée à partir des données zootechniques en sommant les besoins d'entretien, de production laitière et de gains de poids d'après les propositions de Hoden et al (1986). Les besoins relatifs au pâturage ont été estimés à $20 \%$ des besoins d'entretien. L'énergie apportée par les concentrés a ensuite été soustraite du total.

\section{Traitement statistique des résultats}

Les données ont été traitées par analyse de la variance à trois critères de classification, à savoir 
l'année (deux niveaux), le système de pâturage (deux niveaux) et la modalité de pâturage (trois niveaux). Les années n'ont pas eu d'effet significatif de telle sorte que, dans les tableaux présentant les résultats, les moyennes et écarts types des effets «année" ne sont pas donnés. II n'y a pas eu d'interaction entre les effets «année et système» et "année et modalités". Les données relatives aux hauteurs d'herbe dans le pâturage en rotation ont également été traitées selon la procédure General Linear Model du Sas (1985) en utilisant les critères de classification suivants : année, modalité de pâturage, parcelle, cycle et les interactions.

\section{RÉSULTATS}

\section{Déroulement du pâturage}

Les conditions climatiques de première année ont été caractérisées par un temps humide et froid au printemps suivi par un temps sec et chaud, ce qui n'a pas été favorable à une bonne croissance de l'herbe. En fin de saison de pâturage, il n'a pas été possible de garder les animaux sur les parcelles dans le pâturage continu. Les vaches ont été sorties les 2 dernières semaines de l'essai, soit au début du mois d'octobre dans les traitements 4 et 5 et pendant les 4 dernières semaines, soit lors de la deuxième quinzaine du mois de septembre et au début du mois d'octobre dans le traitement 6 . La décision de retirer les animaux des parcelles a été prise lorsque les hauteurs d'herbe étaient inférieures à $3,90 \mathrm{~cm}$ et que la production laitière avait chuté de plus de $1 \mathrm{~kg}$ par semaine. Dans la présentation des résultats, les données correspondant aux périodes de retrait des animaux ont été écartées. En deuxième année, le mois de mai a été humide et froid, le mois de juillet pluvieux et le mois d'août sec et chaud. L'essai a été écourté d'une semaine pour le traitement 6 . Le nombre de jours de pâturage calculés sur la durée expérimentale a été de 560 jours dans le traitement 1,700 jours dans les traitements 2 et 3,532 jours dans le traitement 4,665 jours dans le traitement 5 et 613 jours dans le traitement 6 .

\section{Aspects végétaux}

Le tableau II donne les teneurs en matière sèche, en cellulose brute et en matières azotées totales ainsi que les productions primaires et les hauteurs d'herbe. Les traitements n'ont pas modifié les teneurs en matières azotées et en cellulose brute de I'herbe. La figure 1 montre que la teneur en matières azotées a peu varié au cours de la saison. La production primaire a été en moyenne de l'ordre de $9340 \mathrm{~kg}$ de matière sèche par hectare. L'augmentation du chargement par la fumure azotée a entraîné une amélioration de la production primaire de $16,3 \%$ ( 8781 versus $10216 \mathrm{~kg}$ de matière sèche par hectare), l'augmentation ayant été plus importante dans le système continu $(24,8 \%)$ que dans le système en rotation $(10,3 \%)$. Comme des méthodes différentes ont été employées pour déterminer les productions primaires dans les deux systèmes de pâturage, il n'est pas possible de comparer les résultats. Les hauteurs d'herbe ont été en moyenne de $7,74 \mathrm{~cm}$. L'herbe a été plus courte lorsque la fumure azotée a été plus élevée $(6,99$ versus 8,$01 ; p>0,10)$. L'herbe a été significativement plus courte dans le pâturage en continu que dans le pâturage en rotation ( 4,85 versus $10,61 \mathrm{~cm}$; $p<0,001)$. Dans le pâturage en rotation, l'analyse de variance a mis en évidence un effet significatif de la modalité de pâturage et du cycle $(p<0,01)$. Les hauteurs d'herbe ont été plus grandes dans les traitements 1 et 2 que dans le traitement $3(11,15$ et $11,66$ versus $9,02 \mathrm{~cm} ; p<0,05)$. Les hauteurs ont varié en fonction du cycle : elles ont été plus élevées pendant les cycles 2 et 3 que pendant les cycles 1,4 et $5(13,10$ et 14,01 versus $8,10,11,25$ et $7,04 \mathrm{~cm} ; p<$ $0,01)$. Les faibles hauteurs sont dues à une 
Tableau II. Composition chimique ${ }^{\star}$ (teneur en matière sèche et composition de la matière sèche), production primaire et hauteurs de l'herbe*.

$\begin{array}{ccccc}\text { Matiere } & \text { Cellulose } & \text { Matières } & \text { Production } & \text { Hauteurs de } \\ \text { sèche } & \text { brute } & \text { azotées } & \text { primaire } & \text { l'herbe } \\ (\%) & (\%) & (\%) & (\mathrm{kg} M S / \mathrm{ha}) & (\mathrm{cm})\end{array}$

\section{Systèmes de pâturage}

$1-2-3$

4-5-6

Modalités
$1-4$
$2-5$
$3-6$

Traitements

1
2
3
4
5
6

Niveaux de signification

Système

Modalité

Système $x$ modalité
$15,9 \pm 1,96$

$16,7 \pm 2,23$

$20,1 \pm 0,90$
$20,2+0,83$

$23,8 \pm 1,28$

$23,9 \pm 1,30$

$16,1 \pm 2,05$

$16,2 \pm 2,03$

$16,6 \pm 2,36$
$20,0 \pm 0,58$
$20,1 \pm 0,81$
$20,3 \pm 1,12$
$23,8 \pm 1,05$
$24,0 \pm 1,16$
$23,7 \pm 1,60$
$20,0 \pm 0,51$
$20,2 \pm 0,86$
$20,1 \pm 1,27$
$20,0 \pm 0,67$
$20,0 \pm 0,80$
$20,6 \pm 0,95$
$23,7 \pm 1,10$
$23,9 \pm 1,18$
$23,8 \pm 1,62$
$24,0 \pm 1,04$
$24,0 \pm 1,21$
$23,6 \pm 1,67$

$17,0 \pm 2,60$

NS
NS
NS

\begin{abstract}
NS
NS
\end{abstract}

NS
NS
NS $\begin{array}{rr}10633 & 10,6^{a} \pm 2,69 \\ 8045 & 4,8^{b} \pm 0,47\end{array}$

8781

$10216 \quad 7,0^{\mathrm{a}} \pm 2,98$

10281

10274

11343

7281

7764

9089 $11,1^{\mathrm{a}} \pm 2,67$

$11,7^{\mathrm{a}} \pm 2,86$

$9,0^{b} \pm 2,65$

$4,9^{\mathrm{a}} \pm 0,35$

$4,7^{\mathrm{a}} \pm 0,47$

$4,9^{\mathrm{a}} \pm 0,42$

\footnotetext{
* Valeurs moyennes et écart type. ${ }^{a}, b$ Les valeurs affectées d'une lettre différente dans une mème colonne diffèrent statistiquement entre elles au seuil $p<0,05$ ou $p<0,001$. NS : non significatif : ${ }^{\star \star \star} p<0,001$.
}

moindre croissance de l'herbe au mois de mai (temps froid) et au mois de septembre (facteur saisonnier). II y a eu une interaction significative entre les effets cycle et chargement. Pendant les premier et dernier cycles, la hauteur n'a pas été différente selon la modalité de pâturage. Pendant le deuxième et le quatrième cycles, la hauteur de l'herbe a été plus faible dans le traitement 3 tandis que, pendant le troisième cycle, elle a été plus élevée $(p<0,01)$ dans le traitement 2 par rapport aux deux autres traitements $(p<0,01)$. La composition floristique a été peu influencée par le système de pâturage. Parmi les graminées, seule la présence du ray-grass a été plus importante dans le pâturage continu à raison de 44 contre $36 \%$ dans le système en rotation. L'emploi du haut niveau de fumure azotée a réduit la proportion de trèfle blanc ( 8 versus $12 \%$ ).

\section{Aspects animaux}

Les consommations de complément, les productions laitières, les gains de poids et la composition du lait sont indiqués au tableau III. Les vaches pâturant en continu ont reçu $0,23 \mathrm{~kg}$ de complément en moins que celles du pâturage en rotation. Des quantités 
décroissantes ont été distribuées au cours de la saison aux vaches des traitements 1 , 4 et 3,6 , elles ont reçu en moyenne 1,38 et $1,21 \mathrm{~kg}$ par vache et par jour. Une quantité plus élevée, soit en moyenne $4,04 \mathrm{~kg}$, a été distribuée de façon constante aux vaches des traitements 2 et 5 . La production laitière n'a pas été affectée par le système de pâturage $(14,23$ et $14,61 \mathrm{~kg}$; NS). Elle a été supérieure de plus de $1 \mathrm{~kg}$ dans les traitements 2 et 5 comparée à celle des traitements 1 et $4(15,61$ versus $14,56 \mathrm{~kg}$; NS) et des traitements 3 et $6(15,61$ versus $13,12 \mathrm{~kg} ; p<0,05)$, mais la différence a été plus modérée en lait corrigé à $4 \%$ de matière grasse (moins de $0,5 \mathrm{~kg}$ ). Bien que a)

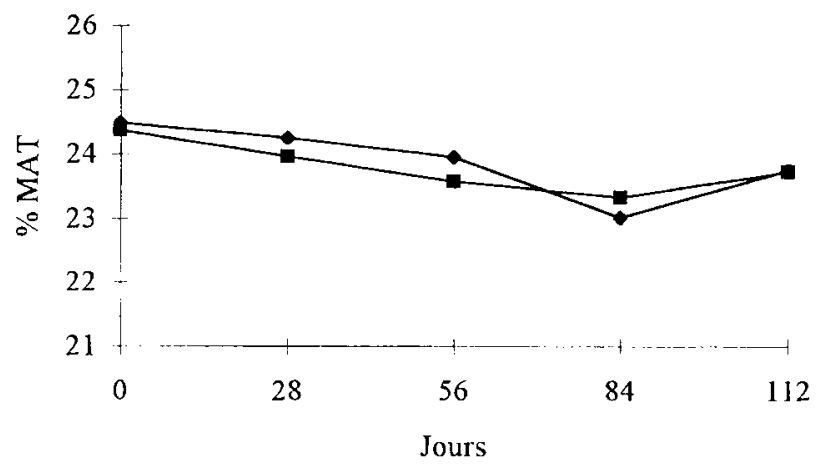

- Traitements 1-2-3 $\downarrow$ Traitements 4-5-6

b)

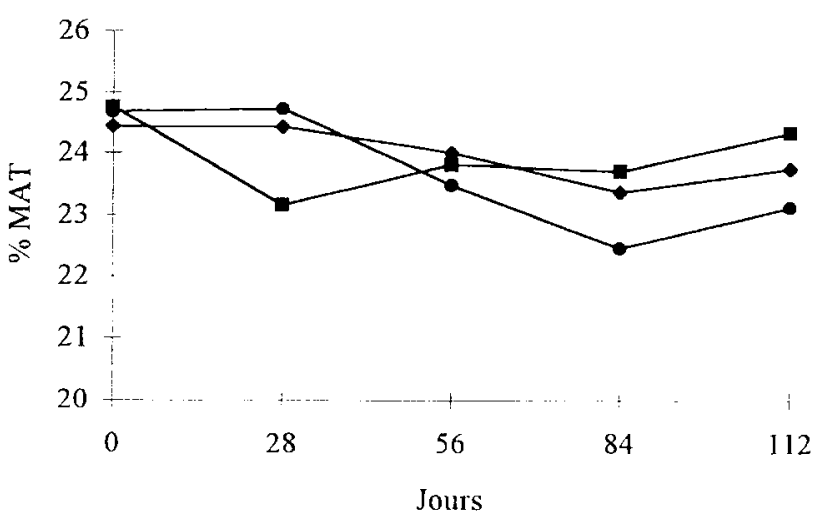

Traitements 1-4
Fig 1. Influence du système de pâturage (a) et de la modalité (b) sur les teneurs en matières azotées totales de l'herbe au cours de la saison de pâturage. 
Tableau III. Consommation de complément, production ${ }^{*}$ et composition du lait ${ }^{\star}$, gain de poids vif ${ }^{*}$ et teneur en urée plasmatique*.

\begin{tabular}{|c|c|c|c|c|c|c|c|}
\hline & $\mathrm{n}$ & $\begin{array}{l}\text { Consommation } \\
\text { complément } \\
(\mathrm{kg} / \mathrm{j} / \mathrm{v})\end{array}$ & $\begin{array}{c}\text { Prod laitière } \\
\text { journalière } \\
\text { (kg/v) }\end{array}$ & $\begin{array}{c}\text { Matière } \\
\text { grasse } \\
(\%)\end{array}$ & $\begin{array}{l}\text { Matière } \\
\text { protéique } \\
(\%)\end{array}$ & $\begin{array}{c}\text { Gain } \\
\text { journalier } \\
(\mathrm{kg} / \mathrm{v})\end{array}$ & $\begin{array}{c}\text { Urée } \\
\text { plasmatique } \\
\text { (mg N/I) }\end{array}$ \\
\hline- & & - & .. & $\ldots$ & ... & - & —. \\
\hline \multicolumn{8}{|c|}{ Système de pâturage } \\
\hline $1-2-3$ & 34 & 2,31 & $14,6^{a} \pm 2,79$ & $3,7^{a} \pm 0,45$ & $3,3^{a} \pm 0,18$ & $0,31^{a} \pm 0,169$ & $209^{a} \pm 33,0$ \\
\hline $4-5-6$ & 34 & 2,08 & $14,2^{\mathrm{a}} \pm 4,13$ & $3,6^{a} \pm 0,45$ & $3,3^{a} \pm 0,21$ & $0,35^{\mathrm{a}} \pm 0,187$ & $270^{b} \pm 27,9$ \\
\hline \multicolumn{8}{|c|}{ Modalités } \\
\hline $1-4$ & 20 & 1,38 & $14,6^{a} \pm 2,57$ & $3,8 a \pm 0,42$ & $3,4^{a} \pm 0,18$ & $0,35^{a} \pm 0,166$ & $231^{a} \pm 41,8$ \\
\hline $2-5$ & 24 & 4,04 & $15,6^{a} \pm 4,40$ & $3,6^{a} \pm 0,43$ & $3,3^{a} \pm 0,17$ & $0,32^{a} \pm 0,183$ & $218^{a} \pm 35,1$ \\
\hline $3-6$ & 24 & 1,21 & $13,1^{a} \pm 2,77$ & $3,6^{a} \pm 0,46$ & $3,3^{a} \pm 0,22$ & $0,33^{a} \pm 0,214$ & $269^{b} \pm 32,7$ \\
\hline \multicolumn{8}{|c|}{ Traitements } \\
\hline 1 & 10 & 1,41 & $14,5^{a} \pm 2,57$ & $3,8^{a} \pm 0,43$ & $3,4^{a} \pm 0,17$ & $0,34 a \pm 0,161$ & $192^{\mathrm{a}} \pm 17,7$ \\
\hline 2 & 12 & 4,18 & $15,6^{a} \pm 3,30$ & $3,5^{a} \pm 0,40$ & $3,3^{a} \pm 0,16$ & $0,33^{a} \pm 0,156$ & $189^{a} \pm 17,7$ \\
\hline 3 & 12 & 1,35 & $13,7^{a} \pm 2,27$ & $3,7^{a} \pm 0,46$ & $3,3^{a} \pm 0,19$ & $0,27^{a} \pm 0,193$ & $246^{b} \pm 27,7$ \\
\hline 4 & 10 & 1,28 & $14,6^{a} \pm 2,71$ & $3,7^{a} \pm 0,72$ & $3,4^{\mathrm{a}} \pm 0,20$ & $0,35^{\mathrm{a}} \pm 0,179$ & $270^{\mathrm{a}} \pm 14,4$ \\
\hline 5 & 12 & 3,89 & $15,6^{a} \pm 5,45$ & $3,6^{a} \pm 0,45$ & $3,4^{a} \pm 0,18$ & $0,32^{a} \pm 0,158$ & $247^{b} \pm 26,4$ \\
\hline 6 & 12 & 1,07 & $12,5^{b} \pm 3,19$ & $3,6^{a} \pm 0,45$ & $3,4^{a} \pm 0,23$ & $0,34^{a} \pm 0,225$ & $292^{\mathrm{a}} \pm 26,1$ \\
\hline
\end{tabular}

Niveaux de signification

Système
Modalité
Système x modalité

$\begin{array}{ll}\text { NS } & \text { NS } \\ * * & N S \\ \text { NS } & \text { NS }\end{array}$

\begin{abstract}
NS
NS
\end{abstract}

NS

$0,34^{\mathrm{a}} \pm 0,225$

\footnotetext{
* Valeurs moyennes et écart type. a,b Les valeurs affectées d'une lettre différente dans une mème colonne diffèrent statistiquement entre elles au seuil $p<0,10, p<0,05$ ou $p<0,001$. NS : non significatif, ${ }^{* *} p<0,10,{ }^{* * *} p<0,001$.
}

l'interaction entre système de pâturage et modalités ait été non significative, la différence entre les traitements 5 et 6 a été particulièrement importante $(15,59$ versus $12,55 ; p<0,05)$.

Les teneurs en matière grasse et protéique et les gains de poids vifs des animaux n'ont pas été influencés par les différents traitements.

La concentration en urée dans le plasma sanguin a été supérieure dans le pâturage continu par rapport au pâturage en rotation et avec les deux traitements recevant la fumure élevée. Une interaction significative a été observée entre système de pâturage et chargement $(p<0,10)$. Dans le pâturage continu, le niveau élevé de concentré a réduit les teneurs en urée mais pas dans le pâturage en rotation. L'évolution globale de la teneur en urée se caractérise par une augmentation au cours de la saison de pâturage (fig 2).

Les vaches soumises au pâturage continu ont présenté des teneurs en urée significativement plus élevées à chaque prélèvement. II en a été de même dans les traitements 3 et 6 . À l'opposé, le haut niveau de concentré a réduit la teneur en urée dans tous les prélèvements. 
a)

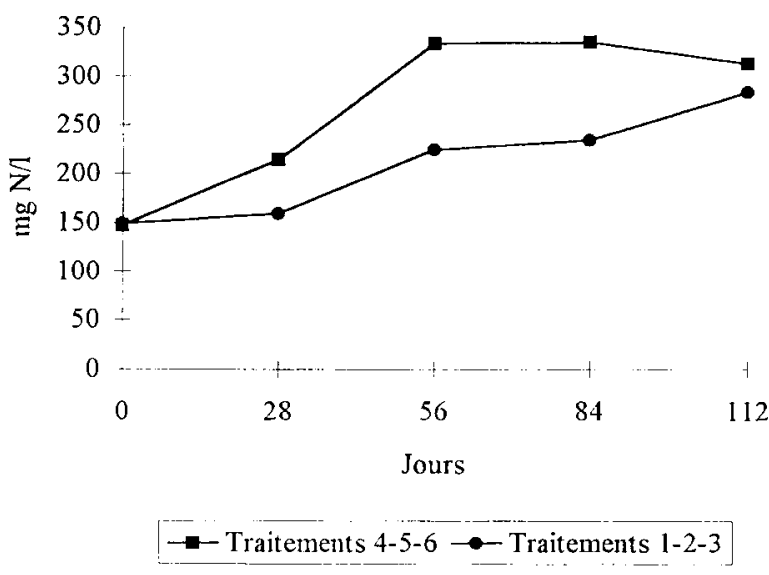

b)

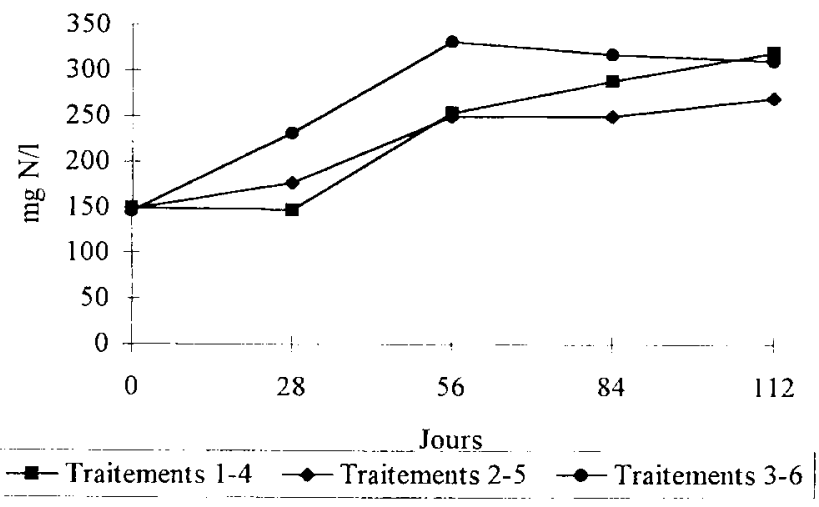

Fig 2. Influence du système de pâturage (a) et de la modalité (b) sur les teneurs en urée plasmatique au cours de la saison de pâturage.

\section{DISCUSSION}

L'augmentation de $1435 \mathrm{~kg}$ de matière sèche d'herbe produite par hectare a été obtenue grâce à un supplément de $112,5 \mathrm{~kg}$ de fumure azotée, ce qui correspond à une réponse de $12,7 \mathrm{~kg}$ de matière sèche par $\mathrm{kg}$ d'azote. Cette réponse a été intermédiaire à celle trouvée entre autres par Jackson et Williams (1979), Prins (1984) et
Frame (1992) respectivement de 4,$5 ; 7,5$ et 15 à $25 \mathrm{~kg}$ de matière sèche d'herbe par $\mathrm{kg}$ d'azote pour des applications ne dépassant pas $400 \mathrm{~kg}$ d'azote/ha. La teneur en matières azotées totales n'a pas été modifiée par l'accroissement de l'engrais azoté alors que l'on observe généralement une augmentation (Demarquilly, 1977). Les résultats observés dans le présent essai peuvent s'expliquer en partie par la réduction de la proportion de trèfle qui était de $8 \%$ 
dans les traitements 3 et 6 alors qu'elle était de $12 \%$ dans les autres traitements. Les hauteurs moyennes d'herbe dans le pâturage en continu mesurées dans cet essai, soit $4,85 \mathrm{~cm}$, ont été inférieures à celles conseillées par Le Du (1980) et Hodgson (1990) pour des vaches laitières, soit respectivement de $6-7 \mathrm{~cm}$ et de $7-9 \mathrm{~cm}$. II n'est pas possible de comparer les productions primaires, ni les hauteurs d'herbe dans les deux systèmes de pâturage en raison des méthodes différentes de mesures employées.

Pendant la période expérimentale d'une durée de 140 jours, l'emploi du système continu ou en rotation a eu peu d'influence sur la production et la composition du lait et sur les gains de poids vif. Ces résultats confirment ceux de Castle et Watson (1975), Mott et al (1980), Carlier et Andries (1981) et Schlepers et Lantinga (1985). La composition du lait a également été peu modifiée dans les essais rapportés par Hood (1974) et Chopping et al (1978). II faut cependant noter une réduction du nombre de jours de pâturage dans le système continu comme l'avaient déjà signalé Limbourg et al (1989). L'emploi du système en rotation a permis de majorer la production laitière de $253 \mathrm{~kg} / \mathrm{ha}$ et a réduit légèrement les gains de poids (tableau IV), tandis que le pâturage continu a entraîné une réduction de 341 UFL/ha correspondant au retrait des animaux en fin de saison, indiquant que le changement adopté était trop élevé pour le système continu. La production de la prairie, exprimée en énergie nette, obtenue pour les 140 jours d'essai, s'élève à 7480 UFL/ha. Limbourg et al (1985) avaient enregistré une productivité de 8295 UFL/ha pour toute la saison expérimentale. Hoden et al (1991) ont obtenu des résultats un peu inférieurs à ceux rapportés dans cet essai (7 $000 \mathrm{UFL/ha)}$ avec un chargement de pâturage élevé.

L'augmentation du niveau de concentré dans les traitements 2 et 5 a amélioré les productions laitières individuelles par rapport à celles des traitements 1 et 4 malgré l'accroissement du chargement. La quan-

Tableau IV. Production de lait, gains de poids vif/ha et productivité nette de la prairie.

$\begin{array}{ccc}\text { Lait } & \text { Gain de poids } & \text { Productivité nette } \\ (\mathrm{kg} / \mathrm{ha}) & (\mathrm{kg} / \mathrm{ha}) & \text { (UFL/ha) }\end{array}$

$\begin{array}{crrr}\begin{array}{c}\text { Systèmes } \\ 1-2-3\end{array} & 9548 & 204 & 7650 \\ 4-5-6 & 9295 & 231 & 7309 \\ & & & \\ \text { Modalités } & 8154 & 195 & 7159 \\ 1-4 & 10929 & 227 & 7111 \\ 2-5 & 9183 & 230 & 8168 \\ 3-6 & & & \\ \text { Traitements } & & 191 & 7369 \\ 1 & 8114 & 230 & 7157 \\ 2 & 10946 & 190 & 8423 \\ 3 & 9584 & 199 & 6949 \\ 4 & 8193 & 223 & 7065 \\ 5 & 10911 & 270 & 7913 \\ 6 & 8782 & & \end{array}$


tité de concentré distribuée a été constante au cours de l'essai dans les traitements 2 et 5 et a diminué dans les traitements 1 et 4 ; ce mode de distribution a pu influencer et augmenter les écarts de production laitière en faveur des traitements 2 et 5 . Les résultats de production primaire d'herbe indiquent des quantités plus faibles d'herbe théoriquement offerte par animal dans les traitements 2 et 5 par rapport aux traitements 1 et 4 (1 804 versus $2195 \mathrm{~kg}$ MS/vache). La quantité d'herbe ingérée par les animaux des traitements 2 et 5 a donc été vraisemblablement plus faible. On sait d'ailleurs que, chez des animaux tels que ceux employés dans le présent essai qui ont des besoins énergétiques modérés et qui reçoivent un niveau élevé de concentré, la distribution de concentré au pâturage provoque une diminution de la consommation d'herbe (Hodgson, 1990). Il est possible d'estimer la quantité d'herbe ingérée à partir des quantités d'herbe offerte et de concentré ingéré d'après les modèles proposés par Meijs et Hoekstra (1984), bien que les méthodes déterminant les quantités d'herbe offerte dans le présent essai soient différentes des leurs. Selon ces modèles, les quantités d'herbe théoriquement ingérées dans les traitements 1 et 4 et dans les traitements 2 et 5 seraient de 11,6 $\mathrm{kg}$ et de $10,0 \mathrm{~kg}$ de matière sèche respectivement. Dans le présent essai, cette réduction de l'ingestion d'herbe dans les traitements 2 et 5 a été compensée par une ingestion supplémentaire de concentré, de $2,66 \mathrm{~kg}$ avec comme conséquence une production laitière supérieure. Comme le supplément de concentré à distribuer était calculé pour couvrir les besoins d'une vache dont le poids et la production laitière correspondaient à la moyenne des traitements, cette production laitière supérieure semble indiquer soit une surestimation des besoins énergétiques des vaches, soit une synergie au niveau de la digestion dans le rumen entre les aliments concentrés, à savoir l'orge et les pulpes séchées et l'herbe pâturée.
Dillon et al (1989) signalent une amélioration de la croissance des microorganismes du rumen lorsque des aliments énergétiques étaient apportés au pâturage. En outre, l'apport de concentré en complément à l'herbe pâturée permet une meilleure utilisation de l'azote soluble contenu dans I'herbe (Van Vuuren, 1993). La production laitière et les gains de poids par hectare ont été augmentés de 34 et de $16 \%$ dans les traitements 2 et 5 par rapport aux traitements 1 et 4 . En revanche, la productivité nette de la prairie n'a pas été améliorée (tableau IV) en raison du taux butyreux moindre et de la plus grande quantité de concentré utilisée. La réponse calculée en terme de kilo de lait par kilo de concentré est égale à 0,39 ; cette réponse a peu varié d'un système de pâturage à l'autre conformément aux résultats obtenus par ArriagaJordan et Holmes (1986). Dans des essais où les niveaux de chargement étaient identiques, Leaver et al (1968), Hoden et al (1975) et Journet et Demarquilly (1979) ont obtenu des réponses de l'ordre de $0,4 \mathrm{~kg}$ de lait $/ \mathrm{kg}$ de concentré. Cependant, exprimée par hectare, la réponse calculée comme étant la différence de production de lait entre les traitements 2 et 5 et les traitements 1 et 4 divisée par la différence de complément distribué entre les mêmes traitements a été plus élevée et correspond à $1,34 \mathrm{~kg}$ de lait/kg de concentré. Cette réponse tient compte de l'augmentation simultanée des niveaux de chargement et de concentré.

Les productions laitières des traitements où la fumure azotée a été augmentée ont été moindres que celles des autres traitements. Les résultats relatifs aux productions primaires d'herbe corroborent ces observations. En effet, dans les traitements 1 et 4 , la production primaire disponible par vache était de $2195 \mathrm{~kg}$ de matière sèche alors qu'elle n'était que de $2043 \mathrm{~kg}$ de matière sèche dans les traitements 3 et 6 . Dans le pâturage en rotation, chaque kilo d'engrais 
azoté supplémentaire a permis d'obtenir 1,25 jour de pâturage supplémentaire, valeur voisine de celle $(1,30)$ obtenue par Limbourg et al (1980) également au pâturage en rotation, tandis que dans le pâturage continu cette réponse n'a été que de 0,72 . La moindre efficacité de l'engrais azoté dans le système continu survient surtout à la fin de la saison de pâturage et s'est traduit par le fait que les animaux du traitement 6 ont dû être sortis de la prairie en fin d'essai, ce qui contribue à expliquer les performances faibles. La réponse permise par kilo d'engrais azoté supplémentaire s'est élevée à $13,1 \mathrm{~kg}$ de lait dans le système en rotation et à seulement $5,2 \mathrm{~kg}$ dans le système continu. Hodgson (1990) considère que, en moyenne, $10 \mathrm{~kg}$ de lait $/ \mathrm{kg}$ d'azote supplémentaire peuvent être obtenus avec une bonne gestion du pâturage.

L'évolution de la concentration en urée plasmatique au cours de la saison de pâturage est un indicateur de l'interaction entre la conduite du pâturage, la composition chimique de l'herbe et la réponse physiologique de l'animal. Les teneurs en urée plasmatique sont également un indicateur des rejets azotés dans un groupe d'animaux (Ciszuk et Gebregziabher, 1994). L'augmentation de la teneur en urée plasmatique au début de l'essai est due au passage d'une ration hivernale équilibrée à une ration constituée principalement d'herbe pâturée contenant un excès de matières azotées en comparaison avec les apports énergétiques. Les teneurs en urée ont augmenté dans tous les traitements au cours de la saison de pâturage tandis que les teneurs en matières azotées de l'herbe n'ont pas été influencées par la saison. Hermansen et al (1994) ont observé une augmentation de la teneur en azote non protéique dans le lait avec l'avancée de la saison de pâturage. Dans le présent essai, ainsi que dans l'essai de Hermansen et al (1994), la tendance saisonnière est confondue avec le stade de lactation, l'excès en matières azotées appor- tées par la ration constituée d'herbe pâturée étant de plus en plus important par rapport aux besoins des animaux, qui diminuent. Les teneurs moyennes en urée plasmatique ont été supérieures à $200 \mathrm{mg} \mathrm{N} / \mathrm{L}$ alors que Vagneur (1992) considère comme optimales pour des vaches laitières en lactation des teneurs s'échelonnant de 92 et $138 \mathrm{mg} \mathrm{N} / \mathrm{L}$. Les valeurs élevées obtenues dans le présent essai sont à mettre en relation avec la teneur élevée en matières azotées de l'herbe. Les teneurs en urée plasmatique des vaches soumises au pâturage continu ont été significativement supérieures à celles observées dans le pâturage en rotation et $\mathrm{ce}$, dès le deuxième mois. L'évolution dans les deux systèmes de pâturage a également été différente : dans le pâturage en rotation, les teneurs ont augmenté de manière constante jusqu'au dernier prélèvement tout en restant inférieures à celles observées en continu tandis qu'on a observé un plateau dans le système continu. Ces différences entre les deux systèmes de pâturage sont difficilement expliquables au vu des teneurs en matières azotées de l'herbe coupée qui n'ont pas été modifiées par le système de pâturage. Cependant la composition de l'herbe coupée ne correspond pas exactement à la composition de l'herbe ingérée par les animaux qui peuvent sélectionner leur ration. Les teneurs plus élevées en urée dans le système continu pourraient être dues à l'ingestion d'herbe plus jeune et plus riche en azote non protéique.

La modalité de pâturage a également influencé les teneurs en urée plasmatique. La teneur en urée plasmatique a été réduite dans le traitement 5 par rapport au traitement 4 car l'apport de concentré supplémentaire dans le traitement 5 a diminué la teneur en matières azotées de la ration ingérée par les animaux. Dillon et al (1989) ont également observé une diminution des teneurs en urée plasmatique chez des vaches laitières au pâturage recevant $3 \mathrm{~kg}$ 
de pulpes séchées mélassées de betteraves ou $3 \mathrm{~kg}$ d'orge. En revanche, dans le système de pâturage en rotation, il n'y a pas eu de différence entre les traitements 1 et 2 : cela pourrait être attribué aux teneurs plus faibles dans ce système de pâturage. Dans les traitements où le niveau de fumure azotée était élevé, les teneurs en urée ont été supérieures, l'augmentation ayant été plus grande dans le système en rotation que dans le système continu. Des résultats semblables ont été rapportés par Astigarraga et al (1993) chez des vaches laitières pâturant une herbe à même disponibilité et produite avec une fumure azotée faible ou élevée. Barabanshchikov (1972) et Bakanov et al (1976) ont également mis en évidence une augmentation de la teneur en urée chez des vaches PieNoir avec l'augmentation de la fumure azotée. Dans le présent essai, il est difficile de mettre en relation l'augmentation des teneurs en urée plasmatique dans les traitements à fumure élevée avec les teneurs en matières azotées de l'herbe coupée qui n'ont pas été modifiées par le niveau de fumure azotée. Il faut cependant souligner que la composition chimique de l'herbe coupée ne reflète pas exactement la composition de l'herbe ingérée par les animaux. L'augmentation des teneurs en urée pourrait, aussi, être liée à une plus grande dégradabilité des matières azotées de I'herbe (Van Vurren et al, 1991) ou à des teneurs plus élevées en azote aminé non protéique ; ces deux paramètres n'ont pas été mesurés dans cet essai.

En conclusion, la production laitière a été peu modifiée par le système de pâturage. L'emploi d'un haut niveau de concentré avec un chargement plus élevé a augmenté la production laitière tandis qu'elle a été réduite lorsque le niveau de fumure azotée était augmenté. La productivité nette de la prairie a été peu influencée par le système de pâturage. La production de lait par hectare a augmenté lorsque le niveau de concentré ou de fumure azotée était accru. La productivité de la prairie a été améliorée uniquement avec le haut niveau de fumure azotée. Le pâturage en rotation, de même qu'un haut niveau de complémentation dans le système continu, permettent de réduire la teneur en urée plasmatique. En revanche, la teneur en urée a été augmentée avec le haut niveau de fumure azotée.

\section{RÉFÉRENCES}

Andries A (1950) L'appréciation dans la pratique de la valeur agricole des herbages, par l'examen de leur composition botanique. Rev Agric 12, 15-19

Arriaga-Jordan CM, Holmes CW (1986) The effect of concentrate supplementation on high yielding dairy cows under two systems of grazing. J Agric Sci Camb 107, 453-461

Astigarraga L, Peyraud JL, Le Bars M (1993) Effect of nitrogen fertilization and protein supplementation on herbage utilization by grazing dairy cows. II. Fecal and urine excretion. In: VIIle Journées des recherches sur la nutrition et l'alimentation des herbivores, 24-25 mars, 49

Bakanov VN, Ovsisher BR, Bondareva NI, Mamaev VA, Filippov VF (1976) Non protein nitrogen and urea in blood and milk of cows in relation to ration composition. Izvestiya Timiryazevskoi Sel'skokhozyaistvennoi Akademii 4, 177-182

Barabanshchikov NV (1972) Milk composition and cheese quality in relation to keeping cows on cultivated grass pastures. Zootekhniya 178, 57-62

Béranger C, Micol D (1981) Utilisation de l'herbe par les bovins au pâturage : importance du chargement et du mode d'exploitation. Fourrages 85, 73-93

Bienfait JM, Gielen M, Limbourg P (1981) Production traditionnelles : exploitation des pâturages par la vache laitière dans les Ardennes belges. Point Vét 1 , 207-213

Carlier A, Andries AP (1981) Exploitation des prairies. 3. Analyse comparative du pâturage continu intensif et du pâturage tournant pour la vache laitière et le bétail à l'engrais. Rev Agric 34, 493-509

Castle ME, Watson JN (1975) Further comparisons between a rigid rotational "Wye college" system and others sytems of grazing for milk production. $J \mathrm{Br}$ Grassld Soc 30, 1-6

Chopping GD, Moss RJ, Goodchiss IK, O'Rourke PK (1978) The effect of grazing systems and nitrogen fertilizer regimes on milk production from irrigated pan- 
gola-couch pastures. In: Proceedings of 12th Australian Society of Animal Production, 229

Ciszuk P, Gebregziabher T (1994) Milk urea as an estimate of urine nitrogen of dairy cows and goats. Acta Agric Scand 44, 87-95

Demarquilly $C$ (1977) Fertilisation et qualitè du fourrage. Fourrages 69, 61-84

Dillon P, Stakelum G, Murphy JJ (1989) The effect of level of herbage intake and concentrate type on rumen fermentation pattern: in situ herbage degradation and blood metabolite levels in lactating dairy cows. In: Proceedings of the 16th International Grassland Congress, Nice, France, 1155-1156

Frame $\mathrm{J}$ (1992) Soil fertility and grass production nitrogen. In: Improved Grassland Management (John Frame, ed), Farming Press books, Royaume-Uni, 101-118

Gately TF, O'Keeffe WF (1984) Review of nitrogen and stocking rate experiments for milk production in Ireland. Ir J Agric Res 23, 11-26

Gielen $M$, Limbourg P, Bienfait JM (1983) Pâturage en rotation ou pâturage continu chez la vache laitière. Agricontact 139, 1-3

Goswami AK, Willcox JS (1969) Effect of applying increasing levels of nitrogen to ryegrass. I. Composition of various nitrogenous fractions and free amino acids. $J$ Sci Food Agric 20, 592-595

Henry RJ, Cannon DC, Winckelman JW (1974) Clinical chemistry: Principles and technics. Harper and Row Publ, New York, $1629 p$

Hermansen JH, Ostersen S, Aaes O (1994) Effect of the levels of $N$ fertilizer, grass and supplementary feeds on nitrogen composition and renneting properties of milk from cows at pasture. J Dairy Res 61 , 179-189

Hoden A, Garel JP, Marquis B, Muller A, Journet M (1975) Complémentation des vaches laitières au pâturage. Bull Tech CRZV Theix 20, 29-35

Hoden A, Micol D, Lienard G, Muller A, Peyraud JL (1986) Interprétation des essais de pâturage avec des bovins : terminologie, modes de calcul, bilans annuels. Bull Tech CRZV Theix 63, 31-42

Hoden A, Muller A, Peyraud JL, Delaby L, Faverdin PM, Peccatte JR, Fargetton M (1991) Pâturage pour vaches laitières. Effets du chargement et de la complémentation en pâturage tournant simplifié. Prod Anim 4, 229-239

Hodgson J (1990) Grazing management. Longman Scientific et Technical, $203 p$

Hood AEM (1974) Intensive set-stocking of dairy cows. J Br Grassid Soc 29, 63-67

Jackson MV, Williams TE (1979) Response of grass swards to fertilizer- $\mathrm{N}$ under cutting or grazing. J Agric Sci $92,549-562$

Journet M, Demarquilly C (1979) Grazing. In : Feeding strategy for the high yielding dairy cow (WHS Bros- ter, ed), Granada Publishing Co, Saint-Albans, 90113

Kibon A, Holmes W (1987) The effect of height of pasture and concentrate composition on dairy cows grazed on continuously stocked pastures. J Agric Sci Camb 109, 293-301

Le Du J (1980) Le pâturage continu : I'expérience anglais. Fourrages 82, 31-43

Leaver JD, Campling RG, Holmes W (1968) Use of supplementary feeds for grazing dairy cattle. Dairy $\mathrm{SCl}$ Abstr 30, 355-361 (Abst)

Lebrun $V$ (1987) Pâturage continu ou pâturage tournant? Fourrages 111, 271-281

Limbourg P, Gielen M, Bienfait JM (1985) Systèmes de pâturage intensif en haute Belgique avec vaches laitières. Bull Rech Agron Gembloux 20, 43-56

Limbourg P, Gielen M, Istasse L, Bienfait JM (1989) Valorisation des pâturages de haute Belgique par la vache laitière à travers deux systèmes d'exploitation différents. In : Proceedings of the XVIth International Grassland Congress, Nice (France), 11411142

Limbourg P, Noirfalise A, Bienfait JM, Gieien M (1980) Fumure azotée et complémentation : deux manières d'augmenter la charge des pâturages par la vache laitière. Ann Méd Vét 124, 497-513

Meijs JAC, Hoekstra JA (1984) Concentrate supplementation of grazing dairy cows. 1 . Effect of concentrate intake and herbage allowance on herbage intake. Grass Forage Sci 39, 59-66

Miettinen PVA, Juvonen RO (1990) Diurnal variations of serum and milk urea levels in dairy cows. Acta Agric Scand 40, 289-296

Mott N, Ernst P, Coenen J, Heiting N (1980) Auf intensivstandweide gleiche leistung, aber weniger arbeit Der Tierzuchter 32, 66-69

Mottar J, De Vilder J (1979) Influence de la composition saline et de la teneur en $\mathrm{N}$ non protéique du lait sur sa stabilité à la chaleur. Rev Agric 32, 803

Oltner R, Wiktorsson $H$ (1983) Urea concentrations in milk and blood as influenced by feeding varying amounts of protein and energy to dairy cows. Livest Prod Sci 10, 457-467

Prins WH (1984) Limits to nitrogen fertilizer on grassland. Neth J Agric Sci 32, 319-321

SAS Institute Inc, 1985. User's guide: statistics, version 5, SAS Inst, Inc, Cary, NC, États-Unis

Schlepers H, Lantinga EA (1985) Comparison of net pasture yield with continuous and rotational grazing at a high level of nitrogen fertilization. Neth J Agric SCi $33,429-432$

Stockdale CR, Trigg TE (1989) Effect of pasture feeding levels on the responses of lactating dairy cows to high energy supplements. Austr J Exp Agric 29, 605-611 
Vagneur M (1992) Biochimie de la vache laitière appliquée à la nutrition. La Dépêche Vétérinaire, supplément technique, 28

Van Vuuren AM, Tamminga S, Ketelaar RS (1990) Ruminal availability of nitrogen and carbohydrates from fresh and preserved herbage in dairy cows. Neth $J$ Agric Sci 38, 499-512

Van Vuuren AM, Tamminga S, Ketelaar RS (1991) In sacco degradation of organic matter and crude pro- tein of fresh grass Lolium perenne in the rumen of grazing dairy cows. J Agric Sci Camb 116, 429436

Van Vuuren AM (1993) Digestion and nitrogen metabolism of grass fed dairy cows. Ph D Thesis, Wageningen, $135 \mathrm{p}$

Vérité R, Journet M, Gueguen L, Hoden A (1980) Vaches laitières. In : Alimentation des ruminants ( $R$ Jarrige,

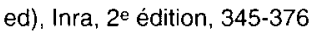

